\title{
7. Die innere Reibung von Argon und ihre Aenderung mit der Temperatur ${ }^{1}$ ); von Hugo Schultze.
}

(Mitgeteilt aus dem Physikalischen Institut zu Halle a/S.)

(Hierza Taf 1.)

\section{Finleitung.}

Lord Rayleigh bestimmte den Reibungscoefficienten des Argons $^{2}$ ), bezogen auf trockene atmosphärische Luft, bei gewöhnlicher Temperatur zu 1,21.3)

Sein Apparat bestand aus zwei durch eine enge Capillare von $1 \mathrm{~m}$ Länge verbundenen Gaspipetten mit Quecksilbermanometern; alle Apparatteile in freier Luft. Beobachtet wurde unter Constanthaltung des Druckes mittels der Gaspipetten die Zeit, in der etwa $100 \mathrm{ccm}$ des Gases bei geringem Ueberdruck aus einem der beiden geteilten cylindrischen Gasbehälter in den anderen übertraten.

Bei dieser Anordnung möchten die Temperaturschwankungen der in freier Luft befindlichen Capillare und der Messgefässe zu merklichen Fehlern Anlass geben. Auch wird die Druckmessung mit einfachen Quecksilbermanometern sowie die Volumenmessung des durchgetretenen Gases mittels der geteilten cylindrischen Glasgefässe keine grosse Genauigkeit bieten.

Es erscheint darnach eine neue Bestimmung des Reibungscoefficienten von Argon wünschenswert. Da überdies bei Argon, als einatomigem Gase, die Aenderung des Reibungscoefficienten mit der Temperatur von grösserem Interesse für die Gastheorie ist als sein Absolutwert, so habe ich auf Anregung und mit freundlicher Unterstützung des Hrn. Prof. Dr. Dorn im Wintersemester 1899/1900 und im Sommersemester 1900 die Reibung des Argons bei verschiedenen Temperaturen untersucht.

1) Auszug aus einer Hallenser Dissertation (1901), in der über verschiedene Einzelheiten nähere Angaben zu finden sind.

2) Lord Rayleigh, Phil. Trans. 186 A. p. 187 ff. 1895.

3) Lord Rayleigh, Proc. Roy. Soc. 59. p. 206. 1896; Report of the British Association (Ipswich) p. 609. 1895. 
Während meiner Untersuchung hat Lord Rayleigh, indem. er $\eta_{\vartheta^{0}}=\eta_{0^{0}}(1+\alpha \vartheta)^{n}$ setzte, den Exponenten $n$ zwischen $15^{\circ}$ und $100^{\circ}$ bestimmt, und zwar zu 0,815. ${ }^{1}$ ) Sein Argon war auf 1-2 Proc. rein. Da er mit fallendem Druck ${ }^{2}$ ) und ohne Vorwärmeröhren an der Capillare arbeitet, bestehen $Z$ weifel über die Temperatur des Gases.

In der vorliegenden Arbeit sind einerseits diese wie die oben angegebenen Fehlerquellen vermieden, andererseits ist der Temperaturcoefficient innerhalb weiterer Grenzen bestimmt worden.

\section{Plan der Untersuchung.}

Zur Bestimmung des Reibungscoefficienten wurde die 'Transpirationsmethode benutzt.

Der Apparat musste ein genaues Arbeiten mit einem geringen Volumen des Gases (etwa $400 \mathrm{ccm}$ ) gestatten, in sich geschlossen und symmetrisch zur Capillare angeordnet sein und eine genaue Constanthaltung des Druckes ermöglichen.

\section{Beschreibung des Apparates.}

Nach einer grossen Anzahl orientirender Vorversuche mit Luft, die mit einer einfacheren Anordnung angestellt wurden, erhielt der Apparat, in vielen Teilen unter Anlehnung an Breitenbach ${ }^{3}$, die auf Tafel I in etwa $1 / 10$ der natürlichen Grösse dargestellte endgültige Form.

Als Gasbehälter dienen auf jeder Seite zwei kugelförmige Gefässe $P, Q$ und $P^{\prime}, Q^{\prime}$, die durch eine Röhre $R$ bez. $R^{\prime}$ von $4 \mathrm{~cm}$ Länge und $0,6 \mathrm{~cm}$ Weite miteinander verbunden sind. Die unteren Kugeln laufen je in eine Röhre von $0,6 \mathrm{~cm}$ lichter Weite aus, die sich in zwei die Hähne $B$ und $C$ bez. $B^{\prime}$ und $C^{\prime}$ tragende Zweige gabelt. Unterhalb der Hähne ist je ein Zweig capillar ausgezogen und dient zum Ablassen des Quecksilbers, von dem anderen, der sich etwa $30 \mathrm{~cm}$ nach unten erstreckt, führt je ein übersponnener langer Gummischlauch zu den Quecksilberbehältern $G$ und $G^{\prime}$.

1) Lord Rayleigh, Proc. Roy. Soc. 66. p. 74. 1900.

2) O. E. Meyer, Pogg. Ann. 148. p. 203. 1873.

3) P. Breitenbach, Wied. Ann. 67. p. 803 ff. 1899. 
Von diesen ist jeder an einem hölzernen Schlitten befestigt, der in einer senkrechten Holzführung gleitet. Ueber ihm befindet sich ein zweiter Schlitten, der nach Feststellung durch die Schraube $F$ bez. $F^{\prime}$ vermittelst einer Messingschraube von $0,2 \mathrm{~cm}$ Ganghöhe und $17 \mathrm{~cm}$ Länge ein langsames Verschieben des unteren Schlittens ermöglicht.

Jede der beiden Kugeln $P$ und $P^{\prime}$ läuft nach oben in eine $0,6 \mathrm{~cm}$ weite Röhre aus, die sich zuerst in eine etwa $50 \mathrm{~cm}$ lange, $0,4 \mathrm{~cm}$ weite, mehrfach gewundene Vorwärmeröhre und dann in ein $0,6 \mathrm{~cm}$ weites T.Stück mit dem Hahn $A$ bez. $A^{\prime}$ fortsetzt. Die hahnlosen Schenkel der T-Stücke stehen durch eingekittete Bleirohre, die mit Aetzkali gefüllten Röhren $H$ bez. $H^{\prime}$ und die Hähne $D$ bez. $D^{\prime}$ mit den Manometern in Verbindung, die anderen Schenkel durch eingekittete Bleirohre mit den Vorwärmeröhren der Capillare.

Als Manometer wurden, da grosse Empfindlichkeit wünschenswert, blosse Wassermanometer aber wegen der Absorption des Argons durch Wasser nicht in Frage kommen konnten, Quecksilberwassermanometer verwandt. Zwei unten durch ein U-Rohr verbundene cylindrische Glasgefässe von $4 \frac{1}{2} \mathrm{~cm}$ lichter Weite und $5 \mathrm{~cm}$ Höhe tragen, das rechte einen $2 \mathrm{~m}$ langen, $0,5 \mathrm{~cm}$ weiten, das linke einen $80 \mathrm{~cm}$ langen, $0,2 \mathrm{~cm}$ weiten Schenkel. Beide Gefässe waren zur Hälfte mit Quecksilber und die obere Hälfte des rechten Gefässes, sowie ein Teil des darüberliegenden Glasrohres mit durch Fluorescein schwach gefärbtem Wasser gefüllt, das bei steigendem Druck an einer Scala in die Höhe stieg. Jeder der mit Wasser gefüllten Schenkel stand oben durch ein eingekittetes Bleirohr mit einem etwa $1 \mathrm{~m}$ tiefer liegenden U-Rohr $K$ bez. $K^{\prime}$ in Verbindung, das mit wassergetränkten Bimssteinstücken gefüllt war. Die Bimssteinstücke waren zuvor, um etwaige schweflige Säure zu entfernen, längere Zeit in destillirtem Wasser gekocht worden. Als Scalen dienten je zwei sorgfältig aneinander gesetzte Spiegelstreifen von $\mathbf{5} \mathrm{cm}$ Breite und je $100 \mathrm{~cm}$ Länge, auf die nach Bunsen mittels Stangenzirkels von einem mit der Teilmaschine hergestellten Originalmaassstabe eine Millimeterteilung abgetragen war. An jedem Manometer waren in einigem Abstande übereinander zwei Thermometer zur Bestimmung der Temperatur der Wassersäulen angebracht. 
Geaicht wurden die Manometer in Intervallen von $5-10 \mathrm{~cm}$ der Teilung durch Vergleichen mit einem Quecksilbermanometer, dessen sehr nahe aneinanderliegenden Schenkel $1,5 \mathrm{~cm}$ lichte Weite hatten. Die Höhendifferenz der Kuppen wurde mittels des Kathetometers auf $1 / 50 \mathrm{~mm}$ genau gemessen. Die abgelesenen Manometerstände erhielten eine Temperaturcorrection auf Grund einer besonders entwickelten Formel.

Die angegebenen Manometer gestatten ein sehr genaues Arbeiten.

1. Es steigt, wie auch praktisch durch Auftragen der Quecksilberdrucke und der entsprechenden Höhen auf Coordinaten nachgewiesen wurde, wegen der cylindrischen Form der Behälter und der Röhren die Wassersäule vollständig proportional dem Drucke, sodass man zwischen je zwei geaichten Stellen des Manometers ohne Beschränkung der Genauigkeit interpoliren kann.

2. Die mit ausgekochten Bimssteinstücken gefüllten U-Rohre an den Manometern verhindern, wie fortdauernde Beobachtungen bei den Versuchen mit Luft ergaben, merklich ein Verdunsten des Wassers, sodass man für jede Versuchsreihe mit Argon, die 3-4 Tage in Anspruch nahm, eine kurz vorher gemachte Graduirung ohne merklichen Fehler zu Grunde legen konnte.

3. Bei Versuchen mit Luft, wo man zu Beginn jedes Versuches durch Oeffnen der Manometer nach aussen die dem Druck des. Barometers entsprechende Stellung der Wassersäule ablesen konnte, war es wegen der cylindrischen Form aller Teile möglich, die vor längerer Zeit für eine andere O-Lage gemachte Manometergraduirung auf die neue $O$-Lage zu transformiren.

Ist nämlich $h$ die bei der Graduirung vorhandene, $h_{1}$ eine spätere $O$-Lage, so entspricht einer Angabe $h^{\prime}$ des Manometers, der für den $O$-Punkt $h$ ein Quecksilberdruck $P$ äquivalent ist, für den Nullpunkt $h_{1}$ ein Druck:

$$
X=\frac{h^{\prime}-h_{1}}{h^{\prime}-h} P \text {. }
$$

Die Gasbehälter $P, P^{\prime}$ und $Q, Q^{\prime}$ befanden sich, um während der Dauer eines Versuches möglichst constante Temperatur zu haben, je in einem doppelwandigen, mit Wasser ge- 
füllten Kasten aus Messingblech. Dieser war umgeben von einem zweiten ebenfalls mit Wasser gefüllten Messingkasten, der das an jeden Behälter sich anschliessende Schlangenrohr umschloss. Zur Ablesung der in die inneren Kästen getauchten Thermometer waren Glasscheiben in den vorderen Wänden der Kästen angebracht.

An die Capillare schlossen sich an beiden Enden Vorwärmeröhren von $0,4 \mathrm{~cm}$ lichter Weite. Sie waren auf der einen Seite zweimal, auf der anderen Seite dreimal gebogen und 100 bez. $150 \mathrm{~cm}$ lang. Zur Befestigung der Capillare samt den Vorwärmeröhren waren die Enden der letzteren in zwei Durchbohrungen einer starken Messingscheibe mit Ueberwurfschrauben, die mit Asbest gedichtet wurden, eingesetzt. In einer dritten Durchbohrung der Messingscheibe befand sich, in derselben Weise befestigt, ein Thermometer.

Ausserdem trug die Messingscheibe auf der Peripherie drei Durchbohrungen, die über drei entsprechende Schrauben des Siedeapparates geschoben werden konnten. Dicht aufgesetzt wurde die Scheibe auf das Temperaturgefäss, indem nach Zwischenlegung eines Ringes aus Asbestpappe die zu den drei Schrauben gehörigen Schraubenmuttern fest aufgeschraubt wurden.

Zur Herstellung eines Wasserbades von $15^{\circ}$ diente bei den Versuchen mit Luft ein einfacher Weissblechcylinder von $70 \mathrm{~cm}$ Höhe und $10 \mathrm{~cm}$ Weite. Die die Capillare tragende Messingscheibe, die etwas über $10 \mathrm{~cm}$ Durchmesser hatte, wurde auf den Rand des Gefässes aufgelegt.

Bei den Versuchen mit Argon bei gewöhnlicher Temperatur wurde, da nach Füllung des Apparates mit dem Gase ein Wechseln des Temperaturgefässes unmöglich war, der unten beschriebene Siedeapparat mit Wasser angefüllt.

Zur Herstellung der Temperaturbäder von $100^{\circ}$ und $183^{\circ}$ diente der auf Tafel I angegebene Siedeapparat aus hartgelötetem Kupferblech. Der untere Teil hat einen Durchmesser von $25 \mathrm{~cm}$ und eine Höhe von $10 \mathrm{~cm}$. Der darüber befindliche doppelwandige Cylinder ist $60 \mathrm{~cm}$ hoch und innen $6 \mathrm{~cm}$ weit. Die Dämpfe strömen durch die Löcher bei $a$ in den Mantel, verdichten sich im Rückflusskühler $b$ und treten als Flüssigkeit durch die Röhre $e$ in den inneren Raum des Apparates zurück. 
Ueber den doppelwandigen Cylinder ist zur Verminderung der Wärmestrahlung ein innen mit Asbestpappe ausgekleideter, aussen hochglanzpolirter Messingcylinder geschoben.

Zum vollständigen Schutze der übrigen Apparatteile vor Wärmestrahlung diente der den Siedeapparat von drei Seiten umgebende, beiderseits mit Stanniol bekleidete Pappschirm $S$.

Um den Apparat evacuiren und mit Gas füllen zu können, setzt sich an den Hahn $\boldsymbol{E}$ ein T-Stück, dessen einer Schenkel durch Bleirohr und dickwandigen Gummischlauch mit dem Hahn $E^{\prime}$ und dessen anderer Schenkel durch eingekittetes Bleirohr mit der Quecksilberluftpumpe und dem Gasometer in Verbindung gebracht werden kann.

Um Anfang und Ende der Versuche zur genauen Zeitablesung zu markiren, diente eine gewöhnlicher Klingelapparat, dessen Schaltung aus Tafel I erhellt.

III. Vorbereitung des Apparates zu den Versuchen.

Alle Glasteile des Apparates wurden nach folgendem von der Physikalisch-Technischen Reichsanstalt angegebenen Recept ${ }^{1}$ ) gereinigt:

1. Benzin, absoluter Alkohol (zur Beseitigung von Fett) in Perlen langsam durchgesaugt, 2. Königswasser, 3. übermangansaures Kali, 4. Wasser (längere Zeit), 5. trockene Luft (mittels Strahlpumpe mehrere Stunden) durchgesaugt.

Nach jeder der ersten Operationen wurde mit destillirtem Wasser gespült.

Die Capillare wurde nach Anschmelzen von kurzen Glasröhren an jeder Seite sorgfältig gereinigt und getrocknet; dann wurden die ebenso behandelten Vorwärmeröhren angeschmolzen. Das Glasblasen geschah durch ein Chlorcalciumrohr.

Die Glashähne wurden mittels einer Mischung von Wachs und Vaselin gedichtet.

An den Kittstellen wurde das Bleirohr, das sich gerade in das Glasrohr hineinschieben liess, mit Siegellack eingekittet und darüber Klebwachs fest angedrückt. Siegellack allein erbält leicht feine Sprünge, hält daher allein nicht dicht.

1) Zeitschr. f. Instrumentenk. 16. p. 143. 1896. 
Der Apparat wurde durch längeres Stehenlassen bei Unterwie bei Ueberdruck auf die Dichtigkeit aller Teile geprüft. Nachdem solche erreicht war, wurde der Apparat zuerst durch mehrmaliges Einsaugen trockener Luft durch Heben und Senken der Quecksilberbirnen, dann im Laufe der Versuche verschiedentlich durch Auspumpen mit der Ge issler'schen Quecksilberpumpe und langsames Wiedereinlassen trockener Luft getrocknet.

Zwei mit Natronkalk und Phosphorpentoxyd gefüllte Trockenröhren mit davorgelegtem Watterohr befanden sich während der Versuche mit Luft beständig vor dem freien Schenkel des T-Stückes unter $E$.

Die Prüfung auf Dichtigkeit wurde im Laufe der Untersuchungen verschiedentlich wiederholt.

Die Manometer wurden, abgesehen von mehreren Graduirungen bei den Vorversuchen zur Feststellung der Genauigkeit der Manometerangaben, einmal zu Anfang und einmal während der Versuche mit Luft, sowie je einmal vor den beiden Versuchsreihen mit Argon graduirt.

Die Angaben des Quecksilbermanometers wurden auf $0^{\circ}$ reducirt. Die Barometerablesungen wurden corrigirt. Zur Zeitmessung diente bei den Versuchen mit Luft ein Fünftelsecunden zeigender, durch Vergleichen mit einer Normalpendeluhr geaichter Chronograph, bei den Versuchen mit Argon ein halbe Secunden schlagender Chronometer.

Die Thermometer für gewöhnliche Temperaturen, mit Ausnahme der an den Manometern angebrachten, bei denen es nur auf relative Angaben zu den Ablesungen bei den Graduirungen ankam, und bei denen grosse Genauigkeit nicht erforderlich war, wurden im Wasserbade mit dem hier calibrirten Normalthermometer des Institutes verglichen.

Die Siedetemperatur des Wassers wurde bei den Versuchen mit Luft aus dem Barometerstand bestimmt.

Bei den Versuchen mit Argon wurde der Siedepunkt des Wassers an einem Thermometer abgelesen, dessen Siedepunkt kurz vorher mittels der Cavendishröhre aus dem Barometerstand ermittelt war.

Die Siedetemperatur des Anilins wurde an zwei in der Physikalisch-Technischen Reichsanstalt geaichtenStabthermometern abgelesen. 


\section{Ausmessung der Capillare.}

$\mathrm{Zu}$ den endgültigen Messungen wurde durch Calibriren mit Quecksilberfäden und mikroskopische Messungen aus einer Anzahl von Capillaren ein etwa $55 \mathrm{~cm}$ langes Stück einer sehr engen Capillare von ziemlich gleichmässigem Caliber und kreisförmigem Querschnitt ausgewählt.

Die Capillare erhielt, um später ohne Aenderung ihrer Dimensionen die Vorwärmeröhren ansetzen zu können, an jedem Ende ein etwa $6 \mathrm{~cm}$ langes Stück $0,4 \mathrm{~cm}$ weites Glasrohr angeschmolzen.

Die Ausmessung geschah nach folgendem Gesichtspunkte. Bei gleichmässigem Caliber würde der Quotient $L / R^{4}$ dem „Reibungswiderstand" entsprechen, falls $L$ die Länge, $R$ den Radius der Capillare bedeutet. Bei ungleichmässigem Caliber ist statt dessen zu setzen $\sum l / r_{s}^{4}$, wenn man nämlich annimmt, dass für die kleine Länge $l$ der Radius $r_{s}$ merklich constant ist. Der reciproke Wert des "Reibungswiderstandes" ergiebt das in der Formel für den Reibungscoefficienten vorkommende $R^{4} / L$.

Nicht weit von den beiden Schmelzstellen wurden auf der Capillare mit Flusssäure die Marken I und II eingeätzt, deren Entfernung mittels horizontal gelegten Kathetometers unter Vergleichung mit einem von dem Normal-Aichamt von Millimeter zu Millimeter geaichten Normalmaassstab aus Messing mit eingelegter Silberteilung mikroskopisch bestimmt wurde. Die Temperatur des Maassstabes wurde an einem daneben gelegten corrigirten Thermometer abgelesen.

Sodann wurde die Capillare ebenfalls mit horizontal gelegtem Kathetometer unter steter Controle der Temperatur mittels eines Quecksilberfadens von nicht ganz $2 \mathrm{~cm}$ Länge von $2 \mathrm{zu} 2 \mathrm{~cm}$ calibrirt. Die Marken I und II lagen innerhalb des calibrirten Teiles; die Lage der Marke II wurde mit abgelesen.

Nach Reduction der Längen des Calibrirungsfadens auf ein und dieselbe Temperatur wurde die Calibrirung mit Angabe der Marke II auf Millimeterpapier aufgetragen. Die reducirten Längen des Fadens an den einzelnen Stellen waren: 


$\begin{array}{lllll}1,919 & 1,922 & 1,929 & 1,929 & 1,922 \\ 1,933 & 1,954 & 1,967 & 1,969 & 1,965 \\ 1,966 & 1,956 & 1,948 & 1,952 & 1,950 \\ 1,943 & 1,932 & 1,921 & 1,920 & 1,918 \\ 1,917 & 1,915 & 1,919 & 1,923 & 1,918 \\ 1,916 & & & & \end{array}$

Die Capillare wurde dann zweimal mittels eines möglichst langen Quecksilberfadens, dessen Lage auf Marke II bezogen wurde, ausgewogen.

Die Längenmessungen wurden unter den angegebenen Maassregeln vorgenommen, die Menisken berücksichtigt (Formel vgl. unten).

Die Masse jedes Quecksilberfadens wurde durch Doppelwägungen, zur Controle zum Teil an zwei Waagen, unter Berücksichtigung des Luftauftriebes im geschlossenen Wägeglas bestimmt. Die O-Lagen wurden vor und nach jeder Wägung bestimmt, das Wägeglas vor jeder Wägung mit einem Leinentuch gut abgerieben und zur Zerstörung etwaiger elektrischer Ladungen leicht durch eine Spiritusflamme gezogen. Angegriffen wurde das Wägeglas, um Niederschlag von Feuchtigkeit zu vermeiden, mit Lederhandschuhen. Nach jedem Oeffnen des Waagekastens wurde mit den Beobachtungen einige Minuten gewartet.

So erhielt man für zwei genau bestimmte Stücke der Capillare einen mittleren Radius nach der Formel

$$
r=\sqrt{\frac{1}{\pi\left[l-\frac{1}{2}\left(h+h^{\prime}\right)\right]}\left[m(1+0,000181 t) 0,07355-\pi \frac{h^{8}-h^{\prime 8}}{6}\right]},
$$

wo $h$ und $h^{\prime}$ die Höhe der Menisken, $l$ die Länge, $m$ die Masse, $t$ die Temperatur des Quecksilberfadens bedeutet.

Von jeder Auswägung wurde die Länge des Quecksilberfadens, vermindert um $1 / 3$ der Höhe des Meniskus an'jedem Ende, in die Calibrirung eingetragen, sie möge mit $L$ bezeichnet werden. $l_{1}, l_{2}, \ldots l_{n}$ seien die hintereinander abgelesenen Längen des Calibrirungsfadens, der, wie schon erwähnt, im Mittel um je $\lambda=2 \mathrm{~cm}$ verschoben wurde. Sind $q_{1}, q_{2}, \ldots q_{n}$ die mittleren Querschnitte der den Fäden $l_{1}, l_{2}, \ldots l_{n}$ entsprechenden, $\lambda \mathrm{cm}$ langen Stücke der Capillare, so müssen, 
da das Volumen $v$ des Calibrirungsfadens bei ein und derselben Temperatur constant ist, die Gleichungen bestehen:

$$
v=l_{1} q_{1}=l_{2} q_{2}=\ldots=l_{n} q_{n},
$$

woraus

$$
q_{1}=\frac{v}{l_{1}}, \quad q_{2}=\frac{v}{l_{2}}, \ldots q_{n}=\frac{v}{l_{n}} .
$$

$L$ umfasse ausser den zu $l_{v}, l_{v+1}, \ldots l_{\mu}$ gehörigen $\lambda$, die Teile $\lambda_{\nu-1}$ und $\lambda_{\mu+1}$ der zu $l_{\nu-1}$ und $l_{\mu+1}$ gehörigen $\lambda$, dann ist das Volumen des zur Auswägung benutzen Quecksilberfadens

$$
V=r^{2} \pi L=\lambda_{\nu-1} q_{\nu-1}+\lambda q_{v}+\ldots \lambda q_{\mu}+\lambda_{\mu+1} q_{\mu+1},
$$

woraus unter Benutzung der Gleichungen (2) folgt:

$$
V=v\left[\frac{\lambda_{\nu}-1}{l_{\nu-1}}+\frac{\lambda}{l_{\nu}}+\frac{\lambda}{l_{\nu+1}}+\ldots+\frac{\lambda}{l_{\mu}}+\frac{\lambda_{\mu+1}}{l_{\mu+1}}\right] .
$$

Hieraus ist $v$ berechenbar. Es ergab sich im Mittel

$$
v=0,00034770 \pm 9 \mathrm{~cm} \text {. }
$$

Mit Benutzung von $v$ folgt weiter

und, indem

$$
q_{1}={ }^{v}, \quad q_{1}=\frac{v}{l_{2}}, \quad \ldots q_{n}=\frac{v}{l_{n}}
$$

$$
q_{1}=r_{1}^{2} \pi, \quad q_{2}=r_{2}^{2} \pi, \ldots \quad q_{n}=r_{n}^{2} \pi,
$$

die Radien $r_{1}, r_{2}, \ldots r_{n}$ für die verschiedenen Caliberintervalle

Für den ,Reibungswiderstand" folgt zunächst für das Stück der Capillare zwischen den beiden Marken:

$$
\frac{\lambda_{1}}{r_{1}^{4}}+\frac{\lambda}{r_{2}^{4}}+\frac{\lambda}{r_{3}^{4}}+\ldots+\frac{\lambda}{r_{n-1}^{4}}+\frac{\lambda_{n}}{r_{n}^{4}},
$$

wo $\lambda_{1}$ und $\lambda_{n}$ die analoge Bedeutung für die Marken I und II haben, wie in (3) $\lambda_{\nu-1}$ und $\lambda_{\mu+1}$ für die Enden des Quecksilberfadens. Die Summe der angegebenen Terme ist:

$$
157969.10^{5} \text {. }
$$

Die Stücke ausserhalb der Marken wurden auf der Teilmaschine mittels Mikroskopes mit Ocularschraubenmikrometer ausgemessen. Das Fadenkreuz des Oculars wurde auf die Marke I bez. II eingestellt, der Schlitten der Teilmaschine um je $\Delta=0,02 \mathrm{~cm}$ verschoben und am Ende jeder Verschiebung der scheinbare Durchmesser, $d_{1}, d_{2}, \ldots d_{n}$, in Scalenteilen des 
Ocularmikrometers ausgemessen. Die wirklichen Durchmesser sind dann

$$
\frac{1}{N} d_{1}, \quad \frac{1}{N}-d_{2}, \ldots \frac{1}{N} d_{n},
$$

falls $N$ den Brechungsindex des Glases bedeutet und das Verhältnis des äusseren Durchmessers der Capillare zum inneren nicht kleiner ist als $N$. Da diese letzte Bedingung für die ausgemessenen Stücke sicher erfüllt war, so verhält sich einfach

woraus

$$
\frac{d_{1}}{d_{s}}=\frac{r_{1}}{\varrho_{s}}
$$

$$
\varrho_{s}=\frac{d_{8}}{d_{1}} r_{1} .
$$

$r_{1}$ ist aber durch die Länge des Calibrirungsfadens in der Gegend der Marke I bez. II durch die Gleichungen (2) und (4) angenähert bestimmt, unter der Annahme nämlich, dass für diese kleine Länge das Caliber der Capillare gleichmässig ist. Damit bekommen wir die einzelnen $\varrho_{s}$ in absolutem Maass und daraus für jedes Ende der Capillaren eine Reihe von Termen

$$
\frac{\Delta}{\varrho_{1}^{4}}, \frac{\Delta}{\varrho_{2}^{4}}, \ldots \frac{\Delta}{\varrho_{s}^{4}}, \ldots \frac{\Delta}{\varrho_{n}^{4}} .
$$

Die Summe dieser Terme ist jenseits von Marke I

$$
1061 \cdot 10^{5}
$$

und jenseits von Marke II

$$
833 \cdot 10^{5}
$$

Addirt man diese Summen zu dem vorher bestimmten „Reibungswiderstand" zwischen den Marken, so ergiebt sich der "Reibungswiderstand“"

$$
\frac{L}{R^{4}}=159863.10^{5}
$$

für die ganze Capillare, woraus das gesuchte $R^{4} / L$ bestimmt ist.

$\mathrm{Zu}$ bemerken ist, dass die Capillare jenseits der beiden Marken zunächst cylindrisch blieb; die Summe der Terme $\Delta / \rho^{4}$ von der Stelle an, wo die kegelförmige. Erweiterung eintrat, war für beide Enden zusammen

$$
418 \cdot 10^{5} \text {, }
$$

also $1 / 382$ vom ganzen „Reibungswiderstand“". 
Die Rechnung ergab:

$$
\log \frac{R^{4}}{L}=0,79675-11
$$

für eine mittlere Temperatur der Capillare von 14,0 $0^{\circ}$.

Bei den Versuchen bei gewöhnlicher Temperatur ist deshalb die Correction wegen Ausdehnung des Glases ohne Bedeutung, bei den Versuchen bei höheren Temperaturen ist die Correction angebracht. Der cubische Ausdehnungscoefficient des Glases wurde nach Dulong und Petit gesetzt:

$$
\begin{aligned}
& 3 \alpha=0,000025 \text { von } 0^{\circ} \text { bis } 100^{\circ} \\
& 3 \alpha=0,000027 \text { von } 100^{\circ} \text { bis } 200^{\circ} .
\end{aligned}
$$

Setzt man die Gesamtlänge der Capillare gleich dern Abstande $L^{\prime}$ der Marken, vermehrt um die Summe sämt,licher $\Delta$, d. i.

$$
L=52,541 \mathrm{~cm},
$$

so bekommt man aus $R^{4} / L$ den mittleren Radius für die ganze Capillare:

$$
R=0,0075717 \mathrm{~cm} \text {. }
$$

Nach O. E. Meyers ${ }^{1}$ ) Versuchen soll mindestens

$$
\frac{L}{2 R}=\frac{3000}{1}
$$

seiu, damit das Poiseuille'sche Gesetz für Gase sicher gilt. Im vorliegenden Falle ist

$$
\frac{L}{2 R}=3469,6 \text {. }
$$

\section{Ausführung der Versuche.}

Der Apparat wurde mit Luft bez. Argon so gefüllt, dass bei Atmosphärendruck der Spiegel des Sperrquecksilbers auf der einen Seite im unteren Teile der Kugel $Q$ bez. $Q$, auf der anderen Seite etwa in der Mitte der Kugeln $Q^{\prime}$ bez. $Q$ stand. Dann wurden die Hähne $E$ und $E^{\prime}$ geschlossen. Nachdem der Apparat einige Zeit zur Erlangung einer constanten Temperatur stehen gelassen war, begannen die Versuche. Der Hahn $A$ bez. $A^{\prime}$, je nachdem das Gas von der rechten Seite des Apparates zur linken oder umgekehrt gedrückt werden

1) O. E. Meyer, Pogg. Ann. 148. p. 44. 1873. 
sollte, wurde geschlossen und durch Heben der Quecksilberbehälter $G$ und $G^{\prime}$ auf jeder Seite Druck gegeben, und zwar auf der Seite, wo der Hahn $A$ bez. $A^{\prime}$ geschlossen war, ein Druck etwas höher als der gewünschte, auf der anderen etwas niedriger als der gewünschte. Das Sperrquecksilber füllte dann die Kugeln $Q$ und $Q^{\prime}$ fast an. Die Druckdifferenz war bei allen Versuchen ziemlich constant gleich $8 \mathrm{~cm}$ Quecksilber. Nachdem das Temperaturbad für die Capillare hergestellt und das Wasser in den Temperaturkästen der Gasbehälter durch Durchblasen von Luft mittels Gummigebläses auf gleichmässige Temperatur gebracht war, wurde $\operatorname{der}$ Hahn $A$ bez. $A^{\prime}$ geöffnet; der Druck auf der $A$ bez. $\mathcal{A}^{\prime}$ entsprechenden Seite fiel, der auf der anderen stieg allmählich. Sobald der gewünschte Druck auf jeder Seite erreicht war, wurde er durch Heben bez. Senken der Füllkugeln mittels der Messingschrauben constant gehalten, was sich sehr genau ausführen liess, weil das Gas durch die sehr enge Capillare schon bei niederer Temperatur äusserst langsam strömte. Der Beginn des Klingelns bezeichnete bei richtiger Schaltung den Augenblick, wo der Spiegel des Sperrquecksilbers den Contact $p_{3}$ bez. $p_{3}^{\prime}$ erreichte und damit den Beginn des Versuches. Mit diesem Moment wurde der Chronograph in Gang gesetzt oder später, bei Verwendung des Chronometers, der damit zusammenfallende halbe Secundenschlag gemerkt, was bei lautem Zählen der halben Secunden vom zweiten Beobachter leicht controlirt werden konnte. Nach Verlauf von 15-20 Min., später von 40-70 Min. wurde unter Hemmung des Chronographen, oder später bei einem bestimmten Secundenschlag des Chronometers der Hahn $B$ bez. $B^{\prime}$ geschlossen und damit der Versuch beendet. Bei entsprechender Stellung der Umschalter zeigte das Aufhören des Klingelns die erfolgte Unterbrechung an.

Das in $P$ bez. $P$ eingetretene Quecksilber wurde durch Oeffnen des Hahnes $C$ bez. $C^{\prime}$ in ein Wägeglas abgelassen und gewogen. Den Zeitpunkt, wo der Contact $p_{3}$ bez. $p_{3}^{\prime}$ vom Quecksilber verlassen wurde, gab wieder der Klingelapparat an.

Die Temperaturen an den Manometern und die Temperaturen der Temperaturbäder wurden zn Anfang und zu Ende jedes Versuches, die des Siedeapparates von $5 \mathbf{z u} 5$ Min. abgelesen. 
Der Barometerstand wurde sofort nach jedem Versuch bestimmt.

Mit Ausnahme der ersten Versuche mit Luft, wurde das im Apparat befindliche Gas abwechselnd von einer Seite zur anderen gedrückt.

Die Constanthaltung des Druckes auf der einen Seite wurde vom Institutsmechaniker Hrn. Böckel, bei einigen Versuchen von Hrn. cand. math. Lindemann besorgt.

\section{Theorie und Berechnung der Versuche.}

Für die Strömung eines Gases durch Capillarröhren findet O. E. Meyer ${ }^{1}$ ) aus den Stokes'schen Gleichungen die angenäherte Formel:

$$
V_{1}=\pi t \frac{p_{1}^{\prime 2}-p_{2}^{\prime 2}}{2.8 . L \eta p_{1}^{\prime}} R^{4}\left[1+\frac{4 \zeta}{R}\right]
$$

worin $p_{1}^{\prime}$ den Druck am Anfang der Röhre, $V_{1}$ das unter diesem Drucke gemessene durchgeströmte Volumen, $p_{2}^{\prime}$ den Druck am Ende der Röhre, $R$ den Radius, $L$ die Länge der Capillare, $t$ die Strömungszeit, $\eta$ den Reibungscoefficienten und $\zeta$ den Gleitungscoefficienten bedeutet.

Hieraus ergiebt sich der Reibungscoefficient:

$$
\eta=\pi t \frac{p_{1}^{\prime 2}-p_{2}^{\prime 2}}{2.8 . L V_{1} p_{1}^{\prime}} R^{4}\left[1+\begin{array}{c}
4 \zeta \\
R
\end{array}\right] .
$$

Für die vorliegende Versuchsanordnung ist $p_{1}^{\prime}$ gleich dem Barometerstand $P$ vermehrt um den Manometerdruck $p_{1}$ und. ebenso $p_{2}^{\prime}=P+p_{2}$, also:

$$
\eta=\pi t \frac{p_{1}\left(2 P+p_{1}\right)-p_{2}\left(2 P+p_{2}\right)}{2\left(P+p_{1}\right)} \frac{R^{4}}{V_{1} L}\left(1+4 \frac{\zeta}{R}\right) .
$$

Ist die Temperatur des betreffenden Gasbehälters, bei der $V_{1}$ gemessen wird, $T$, die der Capillare $T^{\prime}$, so ist vorstehender Ausdruck zu multipliciren mit

$$
\frac{1+\alpha T}{1+\alpha T^{\prime}} .
$$

Um $\eta$ im absoluten Maasse zu erhalten, hat man $t$ in Secunden, $R$ und $L$ in Centimetern auszudrücken. Die auf $0^{\circ}$

1) O. E. Meyer, Pogg. Ann. 127. p. 263 ff. 1866. 
reducirten Quecksilberdrucke $P, p_{1}, p_{2}$ sind in Dynen $/ q \mathrm{~cm}$ umzurechnen. Es ist also zu setzen

$$
p=Q h g \text {, }
$$

wo $Q=13,5957$ das specifische Gewicht des Quecksilbers bei $0^{0}, h$ die Höhe der betreffenden Quecksilbersäule in Centimetern bei $0^{\circ}, g=981,14$ die Schwerebeschleunigung für Halle bedeutet.

$V_{1}$ ist in Cubikcentimetern auszudrücken; da es durch Quecksilber von der Temperatur $T$ ausgemessen ist, so ist:

$$
V_{1}=\frac{M(1+0,000181 T)}{13,5957},
$$

wo $M$ die in Gramm ausgedrückte Masse des Quecksilbers ist. Für die vorliegende Versuchsanordnung gilt also die Formel:

$$
\begin{aligned}
& \eta_{\mathrm{abs} .}=\pi t \frac{\left[p_{1}\left(2 P+p_{1}\right)-p_{2}\left(2 P+p_{2}\right)\right] g 13,5957}{2\left(P+p_{1}\right)} \\
& \frac{R^{4} 13,5957}{L 8 M(1+0,000181 T)} \frac{(1+\alpha T)}{\left(1+\alpha T^{\prime}\right)}\left(1+\frac{4 \zeta}{R}\right) \text {. }
\end{aligned}
$$

Für Luft wurde als Gleitungscoefficient bei Zimmertemperatur der von $\mathrm{Kundt}$ und $\mathrm{Warburg}{ }^{1}$ ) aus Schwingungs. beobachtungen gefundene Wert:

$$
\zeta_{p}=0,00001 \frac{760}{p} \mathrm{~cm}
$$

benutzt; bei 100 und $183^{\circ}$ wurden die von Breitenbach ${ }^{2}$ ) bestimmten angenäherten Werte:

angesetzt.

$$
\begin{aligned}
& \zeta_{p}=0,000013 \frac{760}{\mathrm{p}} \mathrm{cm} \text { bei } 99,15^{\circ}, \\
& \zeta_{p}=0,000012 \frac{760}{p} \mathrm{~cm} \text { bei } 182,4^{\circ}
\end{aligned}
$$

Für Argon, dessen Gleitungscoefficient noch nicht bestimmt ist, wurde die moleculare Weglänge $L$, die theoretisch gleich $\zeta$ ist, in Rechnung gesetzt. 1875 .

1) A. Kundt u. E. Warburg, Pogg. Ann. 155. p. 337 ff. u. p. 525 ff.

2) P. Breitenbach, Wied. Ann. 67. p. 826. 1899. 
Sie ergiebt sich aus den Formeln der kinetischen Gastheorie:

$$
\begin{aligned}
& \left.\eta_{0^{0}}=0,28531 \varrho G L^{1}\right), \\
& \left.G^{2}=3 p \frac{1}{\varrho}^{2}\right) ;
\end{aligned}
$$

für Argon II, wo $\varrho=0,0017787$ und $\eta_{0}$ ( (ohne Berücksichtigung der Gleitung) gleich 2104.10-7, zu

$$
L=0,000010 \mathrm{~cm} \text {. }
$$

Für Argon I, wo $L$ um weniger als 1 Proc. grösser ist, wurde derselbe Wert angesetzt.

Bei der Correction für die Gleitung wurde der mittlere Druck $p$ in der Capillare nach Warburg ${ }^{3}$ ) mittels der Annäherungsformel bestimmt:

$$
p=\frac{P_{0}}{1+\frac{P_{0}-p_{0}}{P_{0}}},
$$

wo $p_{0}=P+p_{1}$ bez. $P+p_{2}$ und $P_{0}=P+p_{2}$ bez. $P+p_{1}$ die Drucke an den Enden der Capillare bedeuten.

Aus den einzelnen Versuchen wurde im Folgenden der Coefficient $\eta^{\prime}$ ohne Berücksichtigung der Gleitung berechnet.

An dem Mittelwert $\eta^{\prime}$ jeder Versuchsreihe wurde dann die Correction wegen des Gleitungscoefficienten angebracht:

$$
\eta=\eta^{\prime}\left(1+\begin{array}{c}
4 \zeta_{p} \\
R
\end{array}\right)
$$

Als Drucke an den Enden der Capillare wurden die Mittelwerte der in jeder Versuchsreihe vorkommenden $P+p_{1}$ bez. $P+p_{2}$ angesetzt, was ohne weiteres geschehen konnte, weil die $P+p_{1}$ bez. $P+p_{2}$ untereinander nur wenig verschieden waren.

VII. Versuche mit atmosphärischer Luft.

In folgender Tabelle gebe ich eine Zusammenstellung der Versuche mit Luft. In der ersten Columne ist angegeben, ab die Luft von rechts nach links $(R)$, oder von links nach rechts $(L)$ durch den Apparat gedrückt worden ist. Im ersten Falle 1899.

1) O. E. Meyer, Kinetische Theorie der Gase, 2. Aufl. p. 189 u. 56.

2) 1. c. p. 24 u. 25.

3) E. Warburg, Pogg. Ann. 159. p. 400. 1876. 
gelten die Ueberdrucke $p_{1}$ und $p_{2}$, im zweiten Falle $\left(p_{2}\right)$ und $\left(p_{1}\right)$. $P$ ist der corrigirte Barometerstand, $T^{\prime}$ und $T$ sind die corrigirten Temperaturen der Capillare und des betreffenden Gas. behälters; $t$ ist die (eventuell corrigirte) Zeit in Secunden, $M$ ist die Masse der dem verdrängten Luftvolumen äquivalenten Quecksilbermenge bei $T^{0}$. In den beiden letzten Columnen stehen die Werte der mit $10^{7}$ multiplicirten Reibungscoefficienten, $\eta^{\prime} 10^{7}$ ohne, $\eta 10^{7}$ mit Berücksichtigung der Gleitung.

Die Versuche bei $15,0^{\circ}$ zeigen wegen zu kurzer Beobachtungszeit mehrfach eine mittlere Abweichung von über $1 / 2$ Proc. Der Mittelwert wird wegen der grossen Anzahl der Versuche doch merklich richtig sein.

Die Luft war durch den in den Trockenröhren vor $E$ befindlichen Natronkalk von Kohlensäure befreit. (Vgl. Tabelle p. 157.)

\section{Darstellung von Argon.}

Das Argon wurde bis auf eine letzte Operation im wesentlichen nach der von Ramsay und Travers ${ }^{1}$ ) gegebenen Methode dargestellt.

„Die Absorption des Sauerstoffs und Stickstoffs wurde in drei Stufen ausgeführt. In der ersten wurde der Sauerstoff durch metallisches Kupfer entfernt; in der zweiten wurde der Stickstoff zweimal über metallisches Magnesium geführt; in der dritten wurde das nun an Argon reiche Gas durch Ueberleiten über eine rotglühende Mischung von Magnesium und wasserfreiem Kalk von den Resten des Stickstoffs und durch rotglühendes Kupferoxyd von Wasserstoff befreit."

Es wurden zwei Portionen Argon dargestellt.

Eine wesentliche Abänderung gegen Ramsay und Travers wurde bei der II. Argondarstellung in der dritten Stufe eingeführt. Um eine vollständige Absorption des Stickstoffs sicher zu erreichen, wurde eine Anordnung getroffen, die gestattete, das Argon mit Hülfe zweier Gaspipetten mehrmals in gleichen Sinne durch den Apparat zu treiben.

Es wurde zu diesem Zwecke ein Glasröhrensystem mit den Hähnen $n, n^{\prime}, v$ und $v^{\prime}$ in der in der Fig. 1 angegebenen Weise in den Apparat eingeschaltet. Wird das Gas von Gas-

1) W. Ramsay u. M. W. Travers, Zeitschr. f. phys. Chem. 28. p. $241 \mathrm{ff} .1899$. 


\begin{tabular}{|c|c|c|c|c|c|c|c|c|c|}
\hline & $T^{\prime}$ & $T$ & $p_{1}\left(p_{2}\right)$ & $p_{2}\left(p_{1}\right)$ & $P$ & $t$ & $M$ & $\eta^{\prime} 10^{7}$ & $\eta 10^{7}$ \\
\hline$R$ & 15,0 & 13,6 & 2,971 & 10,994 & 75,296 & 924,4 & 173,85 & 1799 & 一 \\
\hline$R$ & 15,0 & 13,7 & 2,970 & 10,993 & 75,296 & 920,2 & 172,80 & 1802 & - \\
\hline$R$ & 15,0 & 14,0 & 2,970 & 10,998 & 74,838 & 924,8 & 173,54 & 1802 & - \\
\hline$L$ & 15,0 & 13,6 & 2,953 & 10,939 & 74,911 & 927,2 & 174,39 & 1790 & - \\
\hline$L$ & 15,0 & 11,7 & 2,954 & 10,947 & 75,183 & 922,9 & 172,30 & 1794 & - \\
\hline$L$ & 15,0 & 12,1 & 2,950 & 10,948 & 75,443 & 922,6 & 171,36 & 1807 & 一 \\
\hline$L$ & 15,0 & 13,2 & 2,943 & 10,947 & 75,847 & 924,6 & 172,83 & 1804 & - \\
\hline$R$ & 15,0 & 13,5 & 2,979 & 10,995 & 74,912 & 924,9 & 172,79 & 1808 & - \\
\hline$L$ & 15,0 & 14,3 & 2,945 & 10,944 & 74,648 & 908,8 & 170,19 & 1805 & - \\
\hline$R$ & 15,0 & 14,8 & 2,979 & 10,992 & 75,414 & 929,3 & 174,74 & 1804 & - \\
\hline$L$ & 15,0 & 14,9 & 2,953 & 10,940 & 75,414 & 926,6 & 172,98 & 1812 & - \\
\hline$L$ & 15,0 & 15,8 & 2,944 & 10,946 & 75,072 & 923,3 & 173,53 & 1808 & - \\
\hline$I$ & 15,0 & 11,8 & 2,956 & 10,953 & 75,083 & 908,9 & 169,65 & 1796 & - \\
\hline$R$ & 15,0 & 11,9 & 2,990 & 10,996 & 75,331 & 920,2 & 171,91 & 1797 & - \\
\hline \multirow[t]{2}{*}{$L$} & 15,0 & 12,3 & 2,946 & 10,995 & 74,986 & 908,3 & 169,44 & 1802 & - \\
\hline & 15,0 & - & 2,960 & 10,965 & 75,178 & - & 一 & 1802 & $\left.1811^{1}\right)$ \\
\hline$R$ & 99,8 & 15,9 & 2,972 & 10,980 & 75,513 & 2882,3 & 346,59 & 2190 & - \\
\hline$L$ & 99,8 & 16,3 & 2,944 & 10,937 & 75,430 & 3001,1 & 360,75 & 2190 & - \\
\hline$R$ & 99,8 & 16,4 & 2,974 & 10,979 & 75,514 & 3001,6 & 359,32 & 2203 & - \\
\hline$L$ & 99,5 & 19,4 & 2,931 & 10,925 & 74,755 & 3001,1 & 363,90 & 2194 & - \\
\hline \multirow[t]{2}{*}{$R$} & 99,5 & 20,2 & 2,966 & 10,960 & 74,659 & 3002,3 & 365,64 & 2190 & - \\
\hline & 99,7 & - & 2,957 & 10,956 & 75,174 & - & - & 2193 & 2208 \\
\hline$R$ & 182,7 & 20,3 & 2,976 & 10,961 & 75,057 & 3601,8 & 308,29 & 2551 & - \\
\hline$L$ & 182,6 & $\mathbf{2 0 , 0}$ & 2,929 & 10,936 & 74,952 & 3602,3 & 308,52 & 2554 & - \\
\hline$R$ & 183,2 & 19,6 & 2,977 & 10,962 & 75,232 & 3602,1 & 306,27 & 2560 & - \\
\hline \multirow[t]{2}{*}{$L$} & 183,2 & 20,3 & 2,930 & 10,930 & 74,965 & 3601,6 & 308,10 & 2554 & - \\
\hline & 182,9 & - & 2,953 & 10,947 & 75,052 & - & - & 2555 & 2571 \\
\hline
\end{tabular}

1) Nach Breitenbach's Bestimmung (Wied. Ann. 67. p. 813. 1899) ist, falls die Gleitung berücksichtigt wird:

$$
\eta_{15^{\circ}}=1816 \cdot 10^{-7} \text {. }
$$

Der von Breitenbach bestimmte Reibungscoefficient ist also etwa 1/4 Proc. grösser als der von mir bestimmte. Vielleicht findet diese $\mathbf{A b}$. weichung bei Breitenbach eine Erklärung in der durch die Krümmung, der Capillare verursachten Deformation, die, wenn auch durch Messungen mit Quecksilberfäden nicht merklich nachweisbar, doch den „Reibungs" widerstand" $L / R^{4}$ vergrössert haben mag; Breiten bach würde darnach $R^{4} / L$ etwas zu gross angesetzt haben. 
pipette I nach II gedrückt, so werden die Hähne $n$ und $n^{\prime}$ geöffnet, $v$ und $v^{\prime}$ geschlossen, wird das Gas von II nach I gedrückt, so werden $v$ und $v^{\prime}$ geöffnet und $n$ und $n^{\prime}$ geschlossen. Das Gas strömt dann beide Male in der Pfeilrichtung durch den Absorptionsapparat.

Um die letzten Spuren von Stickstoff, besonders aber etwaige Kohlenwasserstoffe und Wasserstoff aus dem Argon

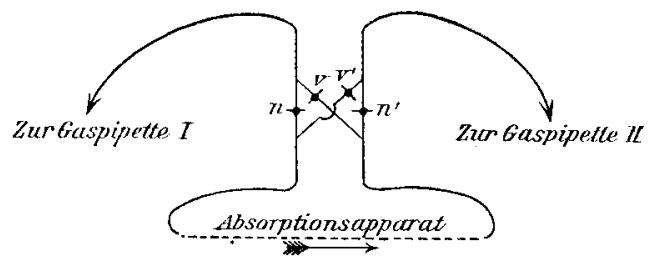

Fig. 1.

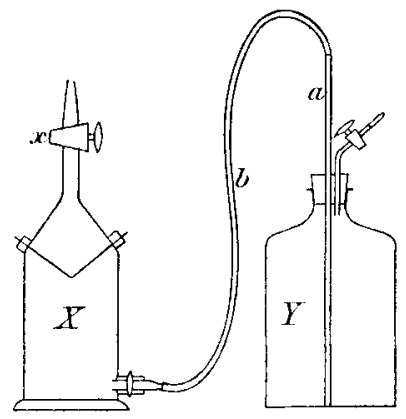

Fig. 2.

zu entfernen, wurde dieses nach Zusatz von reinem Sauerstoff in der in der Fig. 2 angegebenen Funkflasche gefunkt.

Als Sperrflüssigkeit wurde nach $\mathrm{Hempel}^{1}$ ) eine Aetzkalilösung von 120 Gewichtsteilen Aetzkali (das Aetzkali nicht mit Alkohol gereinigt ${ }^{2}$ )) und 80 Gewichtsteilen destillirtem Wasser benutzt.

a) Erste Darstellung von Argon.

Aus der Lösung wurden vor Beginn der Operationen durch Evacuiren der darüker befindlichen Luft etwa absorbirte Gase ausgetrieben. Sodann wurde die in der Fig. 2 gegebene Anordnung hergestellt ( $a$ ist ein Glasrohr, $b$ ein Kautschukschlauch) und durch Heben des Behälters $Y$ die Funkflasche $X$ bis zum Hahn $x$ mit der Aetzkalilösung gefüllt. Nachdem das über dem Hahn befindliche Glasrohr mit destillirtem Wasser angefüllt war, wurde eine Portion reinen Sauerstoffs durch den Hahn $x$ in die Flasche gedrückt.

Hierauf wurde das Argon aus der Gaspipette in die Funkflasche übergefüllt. Sämtliche Verbindungen wurden hierbei

1) W. Hempel, Gasanalyse, II. Aufl. p. 133.

2) l. c. 
mit destillirtem Wasser angefüllt, sodass nur ein Minimum Luft in den Apparat kommen konnte.

Das Gasgemisch wurde an drei Tagen mittels Inductors für $60 \mathrm{~cm}$ Schlagweite mit Turbinenunterbrecher im ganzen etwa 9 Stunden bei $4^{1} / 2 \mathrm{Amp}$. im Primärkreis gefunkt.

Der Sauerstoff wurde dann nach Zusatz von 20 g Pyrogallol in $60 \mathrm{~g}$ destillirtem Wasser $\mathrm{zu}$ der in der Funkflasche befindlichen Kalilauge durch 3 minutiges kräftiges Schütteln entfernt ${ }^{1}$ ) und das übrig bleibende Argon sofort in den Reibungsapparat gebracht (vgl. unten).

\section{b) Zweite Darstellung von Argon.}

Da Kalilauge, falls sie mit Luft in Berührung kommt, vielleicht etwas Stickstoff absorbirt und dieser dann unvermeidlich in kleinen Mengen in das darüber befindliche Argon übertritt, was bei der ersten Darstellung des Gases in der That geschehen war, wurde nach Entfernung absorbirter Gase aus derselben durch Evacuiren des darüber befindlichen Raumes längere Zeit ein starker Sauerstoffstrom hindurchgeleitet und in der Folge dafür gesorgt, dass die Lauge nur mit Sauerstoff in Berührung kam. Die Funkflasche wurde deshalb vor Füllung mit der Aetzkalilösung mit Sauerstoff, der, durch die untere Oeffnung eintretend, die Luft durch den Hahn hinaustrieb, längere Zeit behandelt.

Die Einbringung von reinem Sauerstoff in die Funkflasche geschah in der oben angegebenen Weise.

Zur Ueberfüllung des Argons aus der Gaspipette in die Funkflasche wurden der Capillaransatz der Gaspipette und die über dem Hahn $x$ befindliche Glasröhre der Funkflasche mittels zweier dickwandiger Schlauchstücke und eines T-Stückes, das durch einen Hahn mit der Toeplerpumpe in Verbindung stand, miteinander verbunden. Nach Evacuirung der Zuleitungen wurde der Hahn am T-Stück geschlosssen und das Gas aus der Pipette in den Funkapparat hinüber gedrückt.

Das Glasstück über dem Hahn $x$ war während des Funkens mit destillirtem, ausgekochtem Wasser angefüllt.

Gefunkt wurde etwa 7 Stunden bei $4 \frac{1}{2}$ Amp. im Primärkreis.

1) W. Hempel, l. c. 
Die Pyrogallollösung hatte vor der Benutzung längere Zeit unter dem Recipienten einer Luftpumpe gestanden.

Nach Absorption des Sauerstoffs wurde das übrig bleibende Argon sofort in die Gaspipette zurückgebracht.

\section{Analyse des Argons.}

a) Erstes Argon.

Die spectroskopische Untersuchung des nach Abschluss der ersten Versuchsreihe dem Reibungsapparat entnommenen Argons in der zuvor mit reinem Sauerstoff gespülten Geisslerröhre ergab bei $7 \mathrm{~mm}$ Druck: Argon, Quecksilberdampf, Spuren von Stickstoff. Bei $2 \mathrm{~mm}$ Druck trat der Stickstoff fast ganz zurück.

Das erste Argon enthielt also etwas Stickstoff.

Eine unter Berücksichtigung aller nötigen Maassregeln vorgenommene Dichtebestimmung ergab 19,80, bezogen auf Sauerstoff gleich 16.

Nach Rayleigh ${ }^{1}$ ) wie nach $R$ am say ist die Dichte des reinen, von Neon, Krypton etc. aber noch nicht befreiten Argons 19,94. Das vorliegende Argon enthält also etwa $2^{1} / 3$ Proc. Stickstoff.

b) $Z_{\text {weites Argon. }}$.

Eine Dichtebestimmung des nach dem Funken in die Gaspipette gebrachten Argons ergab 19,89.

Da nicht ausgeschlossen war, dass noch etwas Sauerstoff in dem Gase enthalten war, wurde es vor Ueberfüllung in den Reibungsapparat über glühendes metallisches Kupfer geleitet.

Die spectroskopische Untersuchung einer nach Füllung des Reibungsapparates aus den Zuleitungsrohren entnommenen Quantität Argon ergab bei $10 \mathrm{~mm}$ Druck: Argon, Quecksilberdampf und etwas Stickstoff. Bei 3,5 mm Druck war der Stickstoff nicht mehr zu bemerken.

Die Dichtebestimmung des nach Abschluss der Reibungsversuche aus dem Reibungsapparat entnommenen Argons ergab 19,91 .

Das für die zweite Versuchsreihe verwandte Argon enthielt also nur etwas über $1 / 2$ Proc. Stickstoff.

Die spectroskopischen Untersuchungen wurden von Hrn. Prof. Dorn ausgeführt.

1) Lord Rayleigh, Proc. Roy. Soc. 59. p. 201. 1896. 


\section{Füllung des Reibungsapparates mit Argon.}

a) Zur ersten Versuchsreihe.

Die Funkflasche wurde durch dickwandige Schlauchstücke, ein T-Rohr, das durch einen Glashahn zur Quecksilkerhahnluftpumpe führte, ein mit Natronkalk und Phosphorpentoxyd gefülltes Trockenrohr und dünnes Bleirohr mit dem fleien Schenkel des T-Stückes unter dem Hahn $E$ des Reibungsapparates in Verbindung gebracht. Apparat und Zuleitu igen bis zum Hahn $x$ wurden bis unter $1 / 10 \mathrm{~mm}$ Druck evac uirt, der Hahn des T-Stückes nach der Pumpe geschlossen und durch Oeffnen des Hahnes $x$ das Argon in den Apparat eingelassen, worauf die Hähne $E$ und $E^{\prime}$ geschlossen wurden.

b) Zur zweiten Versuchsreihe.

Die das Argon enthaltende Gaspipette stand mit dem Reibungsapparat durch eine, glühenden Kupferdraht in kleinen Röllchen enthaltende schwer schmelzbare Glasröhre, eine Trockenröhre, den Hahn $A$ und die oben angegebenen Stücke in Verbindung. Das Kupfer war zuvor im Wasserstoffstrom gereinigt und vollständig zu Metall reducirt. Zunächst wurden bei geschlossenem Hahn $A$ der Apparat und die Zuleitungen bis $A$ mittels der Toeplerpumpe scharf evacuirt, der Apparat hierauf abgeschlossen und nach Oeffnen von $A$ die Verbindungen bis zum Hahn $a$ der Gaspipette ausgepumpt. Nachdem die Gasentwickelung in der glühenden Röhre aufgehört hatte; wurden die Hähne zum Apparat wieder geöffnet und das Ganze längere Zeit gut evacuirt. Hierauf wurde der zur Luftpumpe führende Hahn des T-Stückes geschlossen, durch vorsichtiges Oeffnen von $a$ das in langsamem Strom durch die Zuleitungsröhren in den Apparat gebracht und dieser nach genügender: Füllung abgeschlossen. Etwaiger noch vorhandener Sauerstoff war auf diese Weise sicher aus dem Argon entfernt.

\section{Versuche mit Argon.}

Im Folgenden gebe ich eine Zusammenstellung der beiden mit Argon angestellten Versuchsreihen. Hinter jeder Versuchsreihe sind die auf Luft bezogenen Relativwerte von $\eta$ angegeben. Kurz vor der Füllung des Reibungsapparates mit Argon mit Luft angestellte Versuche zur Prüfung des Apparates gaben gute Resultate. Bei der II. Versuchsreihe sind zwei 
über 1 Proc. abweichende Versuche, bei denen irgend ein Febler in der Zeit- oder Druckbestimmung vorlag, nicht mit angegeben.

Der von Lord Rayleigh bestimmte Relatirwert des Reibungscoefficienten, 1,21, ist nur wenig kleiner als der von mir gefundene.

Höchst merkwürdig ist die Thatsache, dass bei dem mit $21 / 3$ Proc. Stickstoff verunreinigten Argon der ersten Versuchsreihe bei gewöhnlicher Temperatur der Reibungscoefficient grösser ausfällt als bei dem nahezu reinen Argon der zweiten Versuchsreihe. Da atmosphärischer Stickstoff einen bedeutend kleineren Reibungscoefficienten hat, sollte man das umgekehrte erwarten.

Unter Benutzung der von mir bestimmten Reibungscoefficienten würde für Argon bei $0^{\circ}$ sein:

I. Versuchsreihe mit Argon.

\begin{tabular}{|c|c|c|c|c|c|c|c|c|c|}
\hline & $T^{\prime \prime}$ & $T$ & $p_{1}\left(p_{2}\right)$ & $p_{2}\left(p_{1}\right)$ & $P$ & $t$ & $M$ & $\eta^{\prime} 10^{7}$ & $\eta 10^{7}$ \\
\hline$L$ & $17,5^{\circ}$ & $18,4^{0}$ & 2,954 & 10,949 & 75,995 & 2400 & 364,45 & 2236 & - \\
\hline$R$ & 17,6 & 18,7 & 2,986 & 10,993 & 75,002 & 2100 & 319,79 & 2235 & - \\
\hline$L$ & 18,2 & 18,9 & 2,953 & 10,949 & 75,062 & 2400 & 366,86 & 2220 & - \\
\hline \multirow[t]{2}{*}{$R$} & 18,3 & 19,2 & 2,986 & 10,991 & 75,086 & 2400 & 366,98 & 2223 & - \\
\hline & $17,9^{\circ}$ & - & 2,970 & 10,971 & 75,036 & - & 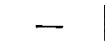 & 2229 & 2241 \\
\hline$L$ & $99,8^{0}$ & $16,7^{\circ}$ & 2,954 & 10,949 & 75,434 & 3600 & 347,14 & 2734 & - \\
\hline$R$ & 99,8 & 18,0 & 2,986 & 10,991 & 75,380 & 3600 & 349,86 & 2728 & - \\
\hline$L$ & 99,8 & 18,6 & 2,952 & 10,947 & 75,353 & 3600 & 350,23 & 2726 & - \\
\hline \multirow[t]{2}{*}{$R$} & 99,8 & 19,1 & 2,985 & 10,990 & 75,304 & $\mathbf{3 6 0 0}$ & 352,62 & 2716 & - \\
\hline & $99,8^{\circ}$ & - & 2,969 & 10,969 & 75,368 & - & - & 2726 & 2740 \\
\hline$R$ & $183,5^{\circ}$ & $19,9^{\circ}$ & 2,985 & 10,990 & 75,045 & 4200 & 289,62 & 3165 & - \\
\hline$L$ & 183,9 & 19,9 & 2,953 & 10,948 & 75,155 & 4200 & 288,75 & 3168 & - \\
\hline \multirow[t]{2}{*}{$R$} & 183,6 & 19,9 & 2,986 & 10,991 & 75,125 & 4200 & 290,57 & 3154 & - \\
\hline & $183,7^{\circ} \mid$ & - & 2,975 & 10,976 & $75,1 \cup 0$ & - & - & 3162 & 3178 \\
\hline
\end{tabular}

Relativwerte bezogen auf Luft:

$\eta(\mathrm{Ar})=1,227 \eta$ (Luft) bei $15,0^{\circ} ; \quad \eta(\mathrm{Ar})=1,241 \quad \eta$ (Luft) bei $99,7^{\circ}$; $\eta(\mathrm{Ar})=1,234 \eta(\mathrm{Lutt})$ bei $183,7^{\circ}$. 
II. Versuchsreihe mit Argon.

\begin{tabular}{|c|c|c|c|c|c|c|c|c|c|}
\hline & $T^{\prime}$ & $T$ & $p_{1}\left(p_{2}\right)$ & $p_{2}\left(p_{1}\right)$ & $P$ & $t$ & $M$ & $\eta^{\prime} 10^{7}$ & $\eta 10^{7}$ \\
\hline$L$ & $15,0^{\circ}$ & $15,4^{\circ}$ & 2,966 & 10,964 & 75,504 & 3060 & 472,68 & 2197 & - \\
\hline$R$ & 15,0 & 15,7 & 2,989 & 11,014 & 75,506 & 2100 & 326,57 & 2192 & - \\
\hline$L$ & 14,4 & 14,4 & 2,967 & 10,967 . & 75,382 & 2400 & 370,49 & 2196 & - \\
\hline$R$ & 14,3 & 14,6 & 2,990 & 11,016 & 75,322 & 2400 & 371,14 & 2201 & - \\
\hline & $14,7^{\circ}$ & - & 2,978 & 10,990 & 75,429 & - & - & 2197 & 2208 \\
\hline
\end{tabular}

\begin{tabular}{l|l|l|l|l|l|l|l|l|l}
\hline$L$ & $99,7^{\circ}$ & $14,4^{0}$ & 2,966 & 10,965 & 75,208 & 3000 & 288,82 & 2719 & - \\
$R$ & 99,7 & 14,8 & 2,965 & 10,963 & 75,017 & 3000 & 290,08 & 2711 & - \\
$L$ & 99,7 & 15,1 & 2,990 & 11,014 & 74,991 & 3000 & 289,53 & 2727 & - \\
\hline & $99,7^{0}$ & - & 2,974 & 10,981 & 75,072 & - & - & 2719 & 2733
\end{tabular}

\begin{tabular}{l|l|l|l|l|l|l|l|l|l}
\hline$L$ & $183,4^{\circ}$ & $14,3^{\circ}$ & 2,967 & 10,965 & $\mathbf{7 4 , 3 9 2}$ & 4200 & 280,81 & 3202 & - \\
$L$ & 183,8 & 14,6 & 2,967 & 10,966 & $\mathbf{7 4 , 2 5 8}$ & $\mathbf{4 2 0 0}$ & $\mathbf{2 7 9 , 9 8}$ & 3212 & - \\
$R$ & 183,9 & 12,9 & 2,990 & 11,017 & 74,468 & 4200 & 279,80 & 3206 & - \\
\hline & $183,7^{\circ}$ & - & 2,975 & 10,983 & $\mathbf{7 4 , 3 7 3}$ & - & - & 3207 & 3224
\end{tabular}

Relativwerte bezogen auf Luft:

$\eta(\mathrm{Ar})=1,220 \eta(\mathrm{Luft})$ bei $15,0^{\circ} ; \quad \eta(\mathrm{Ar})=1,238 \eta$ (Luft) bei $99,7^{\circ}$; $\eta(\mathrm{Ar})=1,252 \eta(\mathrm{Luft})$ bei $183,7^{\circ}$.

XII. Aenderung der Reibung von Argon mit der Temperatur.

Stellt man die Abhängigkeit des Reibungscoefficienten von der Temperatur durch den Factor $(1+\alpha \psi)^{n}$ dar, so ergeben die vorliegenden $\nabla$ ersuche für je zwei aufeinanderfolgende Temperaturen:

$$
\begin{aligned}
& \text { Argon I }\left\{\begin{array}{ccc}
9 & \eta \cdot 10^{7} & n \\
17,9^{0} & 2241 & \\
99,8 & 2740 & 0,8091 \\
183,7 & 3178 & 0,7296
\end{array}\right. \\
& \text { Argon II }\left\{\begin{array}{rrr}
14,7^{\circ} & 2208 & 0,8227 \\
99,7 & 2733 & 0,8119 \\
183,7 & 3224 &
\end{array}\right. \\
& \text { [Luft] } \quad\left\{\begin{array}{rrr}
15,0^{\circ} & 1811 & 0,7675 \\
99,7 & 2208 & 0,7544 \\
182,9 & 2571 &
\end{array}\right.
\end{aligned}
$$


Der von Rayleigh bestimmte Wert für das Intervall von $15-100^{\circ}$ ist also etwas zu klein.

Da der Temperaturcoefficient, wie aus obigen Zahlen hervorgeht, mit der Reinheit des Argons zunimmt, so mag der kleinere Wert zum Teil aus der geringeren Reinheit des von ihm benutzten Argons, zum Teil aber auch aus der unzureichenden Vorwärmung des Gases zu erklären sein.

Die kinetische Gastheorie giebt für die Reibung der Gase die Formel:

$$
\left.\eta=0,30967 \varrho \Omega L^{1}\right),
$$

wo $\Omega$ den arithmetischen Mittelwert der molecularen Geschwindigkeit bedeutet. Da der Reibungscoefficient nur mit der Temperatur, nicht aber mit dem Drucke veränderlich ist, so kann eine Aenderung mit der Temperatur nach obiger Formel nur durch die Factoren $\Omega$ und $L$ bedingt sein, die allein mit der Temperatur veränderlich sind.

In einem Gase, dessen Molecüle unveränderlichen Bestand haben, was bei Argon als einatomigem Gase anzunehmen wäre, sollte theoretisch die Weglänge von der Temperatur unabhängig sein. $\Omega$ ändert sich mit der Wurzel aus $(1+\alpha \vartheta)$, also mïsste sich bei Argon auch der Reibungscoefficient in diesem Verhältnis ändern.

In der That ist jedoch für Argon analog allen bisher untersuchten Gasen $n>0,5$, und zwar wird $n$ bei höherer Temperatur kleiner. $\quad n$ ist sogar noch grösser als bei zweiatomigen Gasen.

Demnach ist auch bei einatomigen Gasen eine Aenderung der Weglänge mit der Temperatur anzunehmen.

Nach der Theorie von Sutherland ${ }^{2}$ ) ist die Weglänge $L$ bei der absoluten Temperatur $\theta$ durch die Formel:

$$
L=L_{0} \frac{1+\alpha C}{1+C: \theta}
$$

mit dem bei der Temperatur des Gefrierpunktes geltenden Werte $L_{0}$ verbunden. $\alpha$ bedeutet den Ausdehnungscoefficienten, $C$ eine von der Cohäsion abhängige Constante.

1) O. E. Meyer, Kinetische Theorie der Gase, 2. Aufl. p. 189. 1899.

2) l. c. p. 168 . 
Die Abhängigkeit der Reibungscoefficienten von der 'Temperatur ist dann dargestellt durch die Gleichung:

$$
\left.\eta=\eta_{0} \frac{1+\alpha C}{1+C: \theta} \sqrt{1+\alpha q^{2}}\right)
$$

welche den bei der absoluten Temperatur, $\theta=\vartheta+1 / \alpha$, geltenden Wert von $\eta$ durch den Wert $\eta_{0}$ ausdrückt, welcher der Temperatur des Gefrierpunktes angehört.

Ich habe aus den drei $\eta_{\vartheta}$ für Argon II $\eta_{0}$ und $C$ nach kleinsten Quadraten berechnet und erhielt:

$$
\eta_{0}=2104 \cdot 10^{-7}, \quad C=169,9 .
$$

Die Zusammenstellung der mit Hülfe dieser Werte berechneten $\eta$ mit den beobachteten ergiebt:

$\begin{array}{rcc}\vartheta & \eta \cdot 10^{7} \text { (berechnet) } & \eta \cdot 10^{7} \text { (beobachtet) } \\ 14,7^{\circ} & 2203 & 2208 \\ 99,7 & 2741 & 2733 \\ 183,7 & 3221 & 3224\end{array}$

Die Formel von Sutherland giebt also eine recht gute Annäherung.

Zum Schluss sei es mir gestattet, meinem hochverehrten Lehrer, Hrn. Prof. Dr. Dorn, für die freundliche Anregung und stete Unterstiitzung bei dieser Arbeit auch an dieser Stelle meinen ergebensten Dank auszusprechen.

1) l. c. p. 221.

(Eingegangen 16. Februar 1901.) 